\title{
Intrahepatic Lymphatic Invasion Independently Predicts Poor Survival and recurrences after Hepatectomy in Patients with Colorectal Carcinoma Liver Metastases
}

\author{
Daniel Jaeck, MD, PhD, FRCS, and Elie Oussoultzoglou, MD \\ Centre de Chirurgie Viscérale et de Transplantation, Hôpital de Hautepierre, Hôpitaux Universitaires de Strasbourg, Avenue \\ Molière, 67200, Strasbourg, France
}

The postoperative recurrence rate after colorectal liver metastases (CLM) resection often exceeds $50 \%{ }^{1}$ Chemotherapy has been associated with a significant decrease of CLM recurrence rate. ${ }^{2}$ Several prognostic factors and clinical scores have been reported from surgical series. These prognostic factors and scores allow improved patient selection for hepatic resection and identify high-risk patients for recurrence who might benefit from adjuvant chemotherapy.

Among the different prognostic factors, presence of hepatic pedicle lymph nodes metastases is now recognized to be one of the strongest risk factor for tumor relapse and for deleterious effect on survival. ${ }^{3,4}$ Interestingly, the survival rate is significantly higher when lymph node involvement is limited to the hepatoduodenal ligament and the retropancreatic area compared to patients with lymph node involvement along the common hepatic artery and around the celiac axis. Specifically, three-year survivors were observed in patients with hepatoduodenal and retropancreatic lymph node metastases while no survival longer than one year was registered for patients with lymph node metastases around common hepatic artery and/or celiac axis. ${ }^{4,5}$ Finally, several factors were associated with hepatic pedicle lymph node

Received August 4, 2007; accepted August 6, 2007; published online: September 27, 2007.

Address correspondence and reprint requests to: Daniel Jaeck, MD, PhD, FRCS; E-mail: Daniel.Jaeck@chru-strasbourg.fr

Published by Springer Science+Business Media, LLC ๔ 2007 The Society of Surgical Oncology, Inc. metastases including the presence of more than three liver metastases, liver metastases located in segment 4 and/or 5, presence of a resectable peritoneal deposit, and poorly differentiated pathological aspect of liver metastases. ${ }^{4}$ Currently, there is no agreement for a systematic hepatic pedicle lymphadenectomy and/or lymph node sampling during liver resection for CLM.

Intrahepatic lymphatic invasion has previously been reported as a pathway of intrahepatic spread in patients with intrahepatic cholangiocarcinoma ${ }^{6}$ and was associated with a worse outcome. For the first time, Sasaki et al. showed that intrahepatic lymphatic invasion constitutes a negative prognostic factor after liver resection for CLM. ${ }^{7}$ The article by Korita et al. published in the Annals of Surgical Oncology investigated the relationship between intrahepatic lymphatic invasion and hepatic lymph node involvement in $\mathrm{CLM}^{8}{ }^{8}$ Once again, the authors underlined the pejorative prognostic value of intrahepatic lymphatic invasion. They retrospectively analyzed the clinical and pathological data of 105 consecutive patients who underwent liver resection for CLM. Specimens were examined to determine the presence or absence of intrahepatic lymphatic invasion using a monoclonal antibody (D2-40) for immunohistochemical staining of intrahepatic lymphatic vessels. They observed a correlation between intrahepatic lymphatic invasion and hepatic lymph node involvement and concluded that intrahepatic lymphatic invasion is an independent prognostic factor for overall and disease-free survival and for intra- and extrahepatic recurrence. 
As pointed out by the authors, their study has several limitations, mainly due to its retrospective design, the small sample size, and the absence of a routine lymphadenectomy. The observed correlation between intrahepatic lymphatic invasion and hepatic lymph node involvement seems plausible and statistically significant. However, this finding should be considered with caution, since lymph nodes dissection was based on their gross appearance and texture on palpation at the time of laparotomy. Indeed, the rate of microscopic hepatic lymph nodes metastases ranged, in several studies, from 11 to $33 \% .^{3-5}$ Therefore, removal of only enlarged or palpable lymph nodes seems insufficient. Selective sampling of the sentinel lymph node or lymphadenectomy based on preoperative imaging particularly on positron emission tomography scan, ${ }^{9,10}$ would be more sensitive than palpation alone. Moreover, the authors proposed immunohistochemical staining to detect intrahepatic lymphatic invasion. However, it would be interesting, as a first step, to compare the sensitivity and the specificity of a standard hematoxylin-eosin histological examination with those of immunohistochemical staining before recommending wider use of the latter technique.

Among the different pathways of intrahepatic invasion by CLM (portal venous invasion, hepatic venous invasion, sinusoidal invasion, bile duct invasion, perineural invasion, stromal invasion) intrahepatic lymphatic invasion is clinically relevant as it is associated with an invariably lethal outcome at three years with disease recurrence. Therefore, the most interesting message of the present study is that in the presence of intrahepatic lymphatic invasion, surgery alone is insufficient to achieve long-term survival even after a curative-intent liver resection for CLM. Further studies are required to determine whether adjuvant chemotherapy may improve outcome for these patients.

The hypothesis that intrahepatic lymphatic invasion is a precursor of hepatic pedicle lymph node metastases needs further investigation. In fact, the reported intrahepatic lymphatic invasion rate of $15.4 \%^{7}$ seems similar to the incidence of hepatic pedicle lymph node involvement $(3-33 \%) .{ }^{4}$ Further evidence of a correlation between intrahepatic lymphatic invasion and hepatic pedicle lymph node metastases is given by the present study. Moreover, the absence of three-year survivors in the case of intrahepatic lymphatic invasion suggests that such invasion may correlate more with distal lymph node metastases (around the common hepatic artery and the celiac axis) or other extrahepatic occult metastases. Identification of predictive factors associated with intrahepatic lymphatic invasion is mandatory. Supposing that intrahepatic lymphatic invasion is an adverse prognostic factor for tumor recurrence after hepatectomy for CLM, a prospective study analyzing the value of adjuvant chemotherapy in such high-risk patients would seem justified.

\section{ACKNOWLEDGEMENT}

The authors express their gratitude to their collaborators Patrick Pessaux, MD, PhD, Edoardo Rosso, MD, Thomas Zacharias, MD.

\section{REFERENCES}

1. Oussoultzoglou E, Bachellier P, Rosso E, et al. Right portal vein embolization before right hepatectomy for unilobar colorectal liver metastases reduces the intrahepatic recurrence rate. Ann Surg 2006; 244:71-9.

2. Kienle P, Koch M, Autschbach F, et al. Decreased detection rate of disseminated tumor cells of rectal cancer patients after preoperative chemoradiation: a first step towards a molecular surrogate marker for neoadjuvant treatment in colorectal cancer. Ann Surg 2003; 238:324-30; discussion 330-1.

3. Elias D, Saric J, Jaeck D, et al. Prospective study of microscopic lymph node involvement of the hepatic pedicle during curative hepatectomy for colorectal metastases. Br J Surg 1996; 83:942-5.

4. Jaeck D, Nakano H, Bachellier P, et al. Significance of hepatic pedicle lymph node involvement in patients with colorectal liver metastases: a prospective study. Ann Surg Oncol 2002; 9:430-8.

5. Laurent C, Sa Cunha A, Rullier E, Smith D, Rullier A, Saric J. Impact of microscopic hepatic lymph node involvement on survival after resection of colorectal liver metastasis. $J$ Am Coll Surg 2004; 198:884-91.

6. Nakajima T, Kondo Y, Miyazaki M, Okui K. A histopathologic study of 102 cases of intrahepatic cholangiocarcinoma: histologic classification and modes of spreading. Hum Pathol $1988 ; 19: 1228-34$.

7. Sasaki A, Aramaki M, Kawano K, Yasuda K, Inomata M, Kitano S. Prognostic significance of intrahepatic lymphatic invasion in patients with hepatic resection due to metastases from colorectal carcinoma. Cancer 2002; 95:105-11.

8. Korita P; Wakai T; Shirai Y, et al. Intrahepatic lymphatic invasion independently predicts poor survival and recurrences after hepatectomy in patients with colorectal carcinoma liver metastases. Ann Surg Oncol 2007; 14. (doi: 10.1245/s10434-0079597-z) .

9. Grobmyer SR, Wang L, Gonen M, et al. Perihepatic lymph node assessment in patients undergoing partial hepatectomy for malignancy. Ann Surg 2006; 244:260-4.

10. Yang YY, Fleshman JW, Strasberg SM. Detection and management of extrahepatic colorectal cancer in patients with resectable liver metastases. J Gastrointest Surg 2007; 11:929-44. 